\title{
Edge-localized mode dynamics and transport in the scrape-off layer of the DIII-D tokamak
}

\author{
J. A. Boedo, ${ }^{\text {a) }}$ D. L. Rudakov, ${ }^{\text {a) }}$ E. Hollmann, ${ }^{a}$ D. S. Gray, ${ }^{a}{ }^{a}$ K. H. Burrell, R. A. Moyer, ${ }^{\text {a) }}$ \\ G. R. McKee, ${ }^{\text {b) }}$ R. Fonck, ${ }^{\text {b) }}$ P. C. Stangeby, ${ }^{\text {c) }}$ T. E. Evans, P. B. Snyder, \\ A. W. Leonard, M. A. Mahdavi, M. J. Schaffer, W. P. West, M. E. Fenstermacher, ${ }^{\text {d) }}$ \\ M. Groth, ${ }^{\text {d) }}$ S. L. Allen, ${ }^{\text {d) }}$ C. Lasnier, ${ }^{d)}$ G. D. Porter, ${ }^{\text {d) }}$ N. S. Wolf, ${ }^{d}$ R. J. Colchin, ${ }^{e}$ L. Zeng, ${ }^{\text {() }}$ \\ G. Wang, ${ }^{f)}$ J. G. Watkins, ${ }^{\text {g) }}$ T. Takahashi, ${ }^{\text {h) }}$ and DIII-D Team \\ General Atomics, P.O. Box 85608, San Diego, California 92186-5608
}

(Received 22 June 2004; accepted 17 May 2005; published online 1 July 2005)

\begin{abstract}
High temporal and spatial resolution measurements in the boundary of the DIII-D tokamak show that edge-localized modes (ELMs) are produced in the low field side, are poloidally localized and are composed of fast bursts ( $\sim 20$ to $40 \mu$ s long) of hot, dense plasma on a background of less dense, colder plasma $\left(\sim 5 \times 10^{18} \mathrm{~m}^{-3}, 50 \mathrm{eV}\right)$ possibly created by the bursts themselves. The ELMs travel radially in the scrape-off layer (SOL), starting at the separatrix at $\sim 450 \mathrm{~m} / \mathrm{s}$, and slow down to $\sim 150 \mathrm{~m} / \mathrm{s}$ near the wall, convecting particles and energy to the SOL and walls. The temperature and density in the ELM plasma initially correspond to those at the top of the density pedestal but quickly decay with radius in the SOL. The temperature decay length $(\sim 1.2$ to $1.5 \mathrm{~cm})$ is much shorter than the density decay length $(\sim 3$ to $8 \mathrm{~cm})$, and the latter decreases with increasing pedestal (and SOL) density. The local particle and energy flux (assuming $T_{i}=T_{e}$ ) at the midplane wall during the bursts are $10 \%$ to $50 \%\left(\sim 1\right.$ to $\left.2 \times 10^{21} \mathrm{~m}^{-2} \mathrm{~s}^{-1}\right)$ and $1 \%$ to $2 \%\left(\sim 20\right.$ to $\left.30 \mathrm{~kW} / \mathrm{m}^{2}\right)$, respectively, of the LCFS fluxes, indicating that particles are transported radially much more efficiently than heat. Evidence is presented suggesting toroidal rotation of the ELM plasma in the SOL. The ELM plasma density and temperature increase linearly with discharge/pedestal density up to a Greenwald fraction of $\sim 0.6$, and then decrease resulting in more benign (grassier) ELMs. (C) 2005 American Institute of Physics. [DOI: 10.1063/1.1949224]
\end{abstract}

\section{INTRODUCTION}

High performance tokamak discharges operate in ELMing $\mathrm{H}$-mode in order to combine high-energy confinement with adequate particle exhaust. However, the edge-localized mode (ELM) instability carries a considerable amount of particles and heat from the pedestal region into the scrape-off layer (SOL) toward the divertor region and other plasma facing components (PFCs), limiting their lifetime and causing the release of impurities into the plasma. According to accepted scalings, type I ELMs are expected to exceed the International Thermonuclear Experimental Reactor (ITER) threshold (40 to $50 \mathrm{MJ} \mathrm{m}^{-2} \mathrm{t}^{-1 / 2}$ ) by factors of 5 or more, ${ }^{1-4}$ resulting in a divertor lifetime of less than one full discharge. On the other hand, recent results ${ }^{5-7}$ indicate that the initial ELM energy scaling laws, showing a linear dependence with pedestal pressure, can perhaps be circumvented if plasma operation is conducted at high density/collisionality. It is, therefore, important to reduce the ELM deposited energy and/or extend its duration to keep the energy deposition below the thresholds. Additionally, ELMs can have a deleteri-

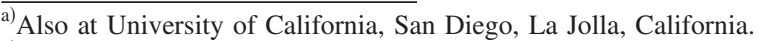

b) Also at University of Wisconsin.

c) Also at University of Toronto, Toronto, Canada.

d) Also at Lawrence Livermore National Laboratory, Livermore, California.

e) Also at Oak Ridge National Laboratory, Oak Ridge, Tennessee.

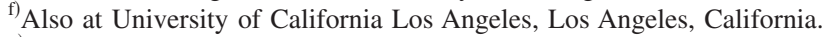

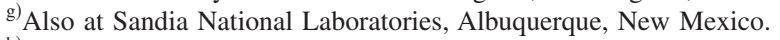

${ }^{\text {h) }}$ Also at Princeton Plasma Physics Laboratory, Princeton, New Jersey.
}

ous effect on confinement by suddenly $(<1 \mathrm{~m} / \mathrm{s})$ collapsing the pedestal region and causing an inward cold pulse. Therefore, it is important to invest a significant effort to study ELMs and the ELM dynamics in the SOL in order to envision ways of controlling their interaction with the PFCs.

A number of theoretical studies ${ }^{8}$ of ELMs have noted that the sharp pressure gradients, and consequent large bootstrap currents in the pedestal region, can destabilize magnetohydrodynamic (MHD) peeling (i.e., edge-localized external kink) and ballooning modes over a wide range of toroidal mode numbers $(n)$. Recent studies ${ }^{9-11}$ have emphasized the complex dual role the bootstrap current plays in the stability physics, on one hand driving peeling modes, while on the other lowering edge shear and opening second stability access to high- $n$ ballooning modes. Field line bending stabilizes long wavelength modes, while short wavelengths are stabilized by a combination of second stability and finite Larmor radius (FLR)/diamagnetic effects, shifting the limiting modes to intermediate wavelengths (typically $n \sim 4$ to 40). These dominant modes are referred to ${ }^{9,10}$ as coupled "peeling-ballooning" modes, and are driven by both parallel current $\left(J_{p e d}\right)$ and the pressure gradient $\left(p_{\text {ped }}^{\prime}\right)$. These intermediate- $n$ peeling-ballooning modes impose constraints on the pedestal height, which are functions of the pedestal width, plasma shape, collisionality, safety factor, and other equilibrium details. Studies of the nonlinear evolution of these peeling-ballooning modes are in their infancy; however, some expected general characteristics of a peeling- 


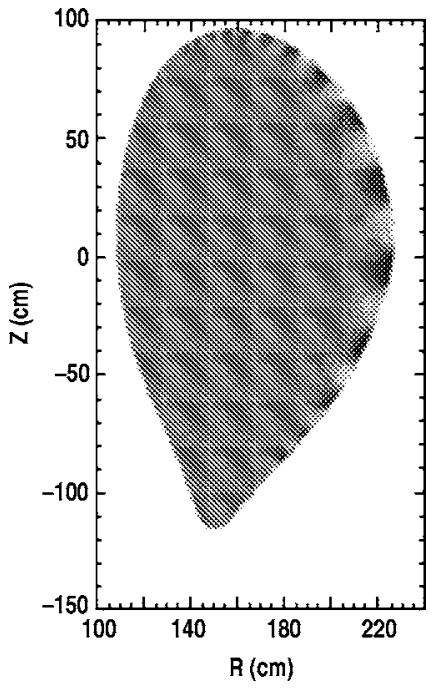

FIG. 1. ELITE calculation showing a poloidal cross section of DIII-D and indicating the peeling-ballooning perturbations to the equilibrium which feature a high $m$ number. The amplitude of the perturbation is greatest at the low field side and rapidly diminished with poloidal angle.

ballooning mode driven ELM crash can be surmised from linear calculations of mode structures along with insight from preliminary nonlinear studies.

A characteristic linear mode structure for an $n=10$ peeling-ballooning mode in a DIII-D plasma, calculated using the ELITE code, ${ }^{10,11}$ is shown in Fig. 1 . The mode has its maximum amplitude on the outer midplane and has very small amplitude on the high field side of the plasma. The mode structure follows field lines and, thus consists of a series of filaments with a length $\sim q R$ along the field line, a poloidal wavenumber of roughly $n q$, and a radial extent somewhat larger than the pedestal width (noting that the local field line curvature should replace $q$ for a more accurate estimate). Preliminary nonlinear studies ${ }^{12}$ suggest that during the ELM, a number of these filaments will grow in the pedestal region and travel across the separatrix into the SOL, carrying particles and heat with them. Series of model equilibria have been constructed for DIII-D discharges, matching the observed values of $B_{t}, I_{p}, R, a$, pedestal density, triangularity and ellipticity, following recently developed techniques. ${ }^{10}$ Pedestal stability boundaries, calculated by ELITE, suggest that the pedestal in these shots (the ones used in this study) is reaching peeling-ballooning stability boundaries when ELMs are observed. This is in agreement with other studies of ELMs in DIII-D, ${ }^{10,11}$ which have consistently found agreement between ELM observations and the peeling-ballooning model.

However, the existing theoretical understanding does not predict the nonlinear phase of the ELM, has just included toroidal rotation, and has yet to include other effects such as poloidal rotation and finite resistivity. ${ }^{12}$ The effect of toroidal rotation implemented on the ELITE model is to slightly narrow the modes radially and extend them poloidally. Therefore, experimental work still remains to be done on providing the theorists with detailed information on ELM genesis, structure and propagation that can be used to improve the models. This paper attempts to provide an answer to some of

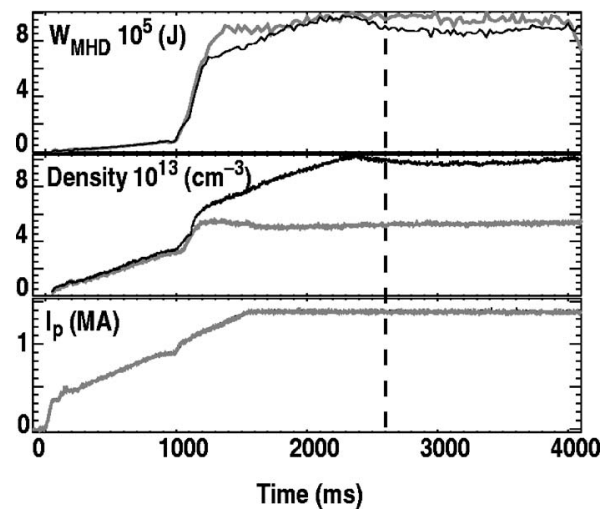

FIG. 2. Time evolution of two H-mode discharges in DIII-D showing from top to bottom: energy content, density and plasma current. The two discharges have different density but are otherwise identical. The probe is inserted at the time marked by the thick vertical lines.

the unknowns such as where the ELMs are forming, their radial and poloidal extent and dynamics once they leave the pedestal.

\section{EXPERIMENTAL SETUP}

Experiments to characterize ELMs on the DIII-D tokamak ${ }^{13}$ were carried out in $\mathrm{H}$-mode discharges featuring type I ELMs with plasma current $I_{p}=1.4 \mathrm{MA}$, toroidal field of $B_{T}=1.7 \mathrm{~T}$ at the axis, $R=1.7 \mathrm{~m}$ and neutral beam heating power of up to $4.5 \mathrm{MW}$. Lower single-null divertor geometry (at the bottom of the vacuum vessel) with ion $\Delta \mathrm{B}$ drift toward the divertor was used. The density was increased in a series of sequential discharges, two of which are shown in Fig. 2, from $\left\langle n_{e}\right\rangle / n_{G W}=0.40$ to 0.8 .

The principal measurements were made by the fast radiometer array ${ }^{14}$ (DISRAD2), a fast scanning probe, ${ }^{15}$ beam emission spectroscopy ${ }^{16}$ (BES), reflectometry ${ }^{17}$ and the divertor current monitor tile array. ${ }^{18}$ The fast scanning probe array features five tips (Fig. 3, upper inset) that sense current $I$, saturation current, $I_{s a t}$, and floating potential, $\Phi_{f}$. The ion saturation current $I_{\text {sat }}$, given by the expression $I_{\text {sat }}$ $=1 / 2 A_{p r} e n_{e} c_{s}=1 / 2 A_{p r} e n_{e}\left[k\left(T_{e}+T_{i}\right) / m_{i}\right]^{1 / 2}\left(T_{e}\right.$ and $T_{i}$ are the ion and electron temperatures respectively, $A_{p r}$ is the tip area and $n_{e}$ is the electron density) and thus the electron density can be extracted if $T_{e}$ is known (assuming $T_{e}=T_{i}$ ). A fast $T_{e}$ measurement ${ }^{19}$ with bandwidth of $\approx 250 \mathrm{kHz}$ is used to resolve the temperature inside the ELMs. All probe measurements are combined to infer the radial particle flux, $\Gamma_{r}$, defined as $\left\langle n_{e} E_{\theta}\right\rangle / B_{\phi}$. The poloidal electric field, $E_{\theta}=\left(V_{f 1}\right.$ $\left.-V_{f 2}\right) / \delta$, for probe tips separated a distance $\delta$; all sampled at a $1 \mathrm{MHz}$ rate. The probe scans horizontally from the outside wall in approximately $250 \mathrm{~m} / \mathrm{s}$ along the path indicated in Fig. 3 (lower inset). The calculation of $E_{\theta}$ assumes that the temperature and sheath drop are identical in both floating potential tips and that the objects under study are larger than the separation between the tips $(4 \mathrm{~mm})$. The last assumption is justified later using BES data. The plasma potential $V_{p}$ was calculated as $V_{p} \approx \Phi_{f}+3.0 k T_{e}$.

The BES system, configured as a $5 \times 6$ fiber array as seen in Fig. 3 (lower inset), was located at the edge of the 


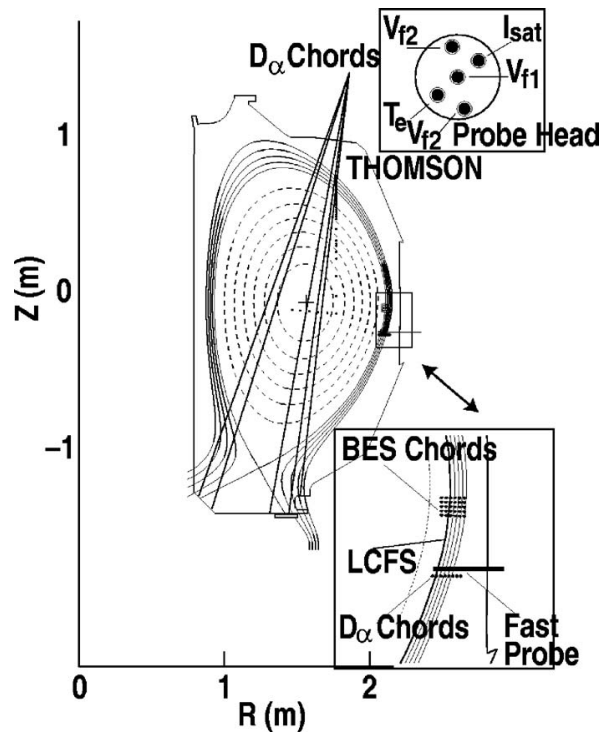

FIG. 3. Poloidal cut of DIII-D showing the magnetic geometry and some relevant diagnostics. Two insets show (top) the scanning probe tip geometry and (bottom) the BES diagnostic geometry. The Thomson scattering tangential chords are indicated as blue dots. Some of the $D_{\alpha}$ chords viewing the divertor floor are also indicated. One of the divertor current monitor tiles is highlighted at the outer strike point.

plasma in the midplane to provide fast $(1 \mu \mathrm{s})$ twodimensional (2-D) imaging of the density. The DISRAD2 $2^{14}$ measures absolutely calibrated photon intensity along 30 poloidally separated view chords, as shown in Fig. 4, at a toroidal angle of $225^{\circ}$ at a rate of $100 \mathrm{kHz}$ and a photon energy response of $1 \mathrm{eV}$ to $5 \mathrm{keV}$. Most of the plasma radiation is in the range 10 to $500 \mathrm{eV}$ (Lyman-a and $\mathrm{C}$ lines), falling well within the DISRAD2 spectral response. To interpret the lineintegrated radiated power measurements, the SOL is broken into seven poloidally separated emission regions and the divertor is broken into three regions (an outer leg, an inner leg, and the PFR, as shown in Fig. 4. The radial boundaries of the

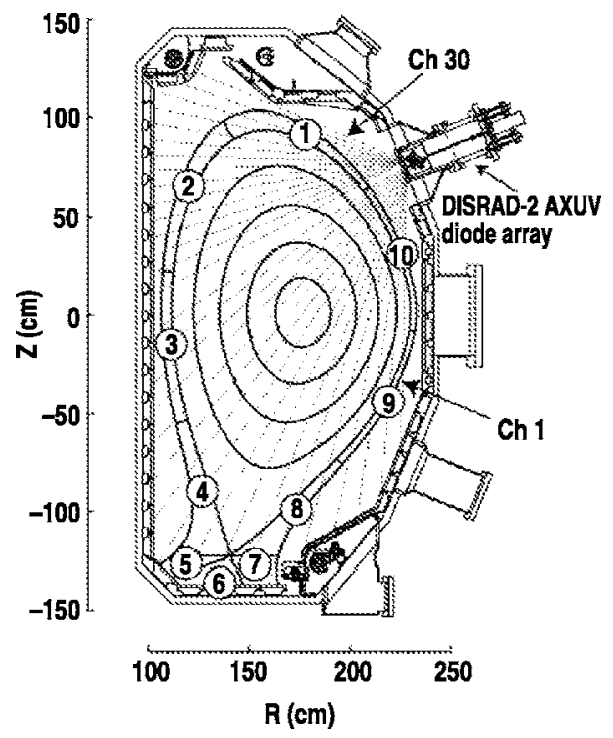

FIG. 4. DISRAD2 viewing geometry showing the 30 view chords and the 10 emissivity regions used to invert the line-integrated data.

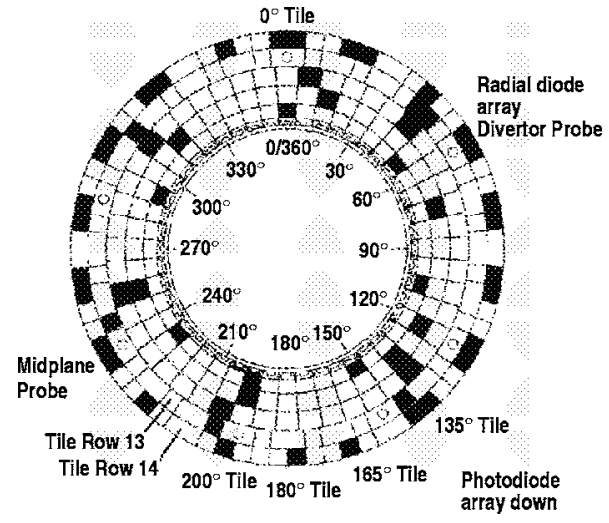

FIG. 5. View of the divertor floor, indicating the current monitor tiles as black blocks and indicating the toroidal location of some of the diagnostics used.

regions are made using EFIT magnetic reconstructions ${ }^{20}$ of the flux surfaces. The line-integrated data are then inverted assuming that the emissivity is constant in each of these regions. Least-squares with constrained positivity is used since the inversion is over-determined (10 regions with 30 data points at each time slice).

Other critical diagnostics are: the reflectometer at DIII-D ${ }^{21}$ that operates at 33 to $55 \mathrm{GHz}$ and can sweep its frequency range in $5 \mu \mathrm{s}$, thus providing fast density profile information, and the divertor current monitor array, shown in Fig. 5, consists of many tiles configured as current monitors with a frequency response of $1 \mathrm{MHz}$. A variety of other diagnostics such as tangential and vertical D-alpha arrays ${ }^{22}$ are also shown in Fig. 3. All the above measurements are mapped onto the magnetic surfaces, displayed in Fig. 3, calculated by the toroidal equilibrium fitting code EFIT. ${ }^{23}$

\section{GENERAL ELM CHARACTERIZATION}

Initially, we will try to shed some light on general questions about ELMs, such as where are they formed, what kind of structure they possess and how they move in the edge/ SOL.

Analysis of the DISRAD2 data ${ }^{14}$ (Fig. 6) shows the in-

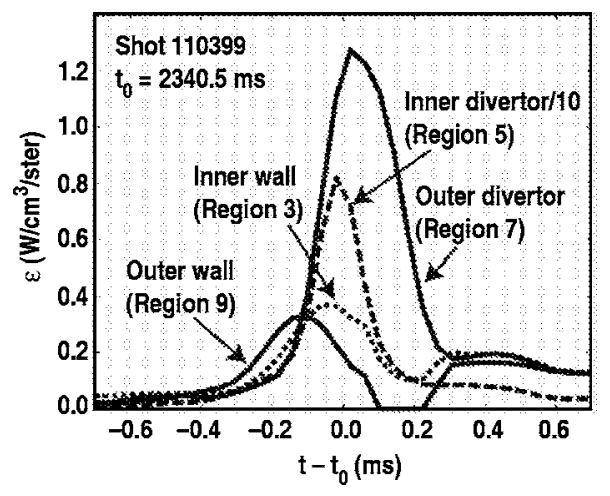

FIG. 6. Inversion of DISRAD2 data from a single ELM (type I) showing radiation peaking first in the outer SOL (region 9), then in the inner SOL (region 3), later at the inner divertor (region 5), and last at the outer divertor (region 7), demonstrating ELM propagation over the top of the plasma into the inner divertor leg. 
tegrated ELM radiation for the various plasma regions indicated in Fig. 4 as a function of time measured from the beginning of an arbitrary ELM or $t_{0}$. The data indicate that the radiation rises first on the outer SOL (regions 9 and 10) indicating low field side ELM formation, it then rises in the inner SOL (regions 2 and 3) and thereafter in the inner and outer SOL divertor regions simultaneously. The risetime of the signals is $\sim 100-200 \mu \mathrm{s}$, consistent with ELM plasma radial propagation as will be discussed later. Additional evidence supporting low field side ELM formation in DIII-D, based on double-null configuration ELM divertor fluxes, has also been recently presented. ${ }^{23}$ Typically, about one-half of the ELM radiation comes from the LFS and HFS SOL regions before rising at the divertor region clearly demonstrating significant ELM radial motion. Particle propagation times between the outer wall and divertor (i.e., parallel propagation) are of the order $100 \mu \mathrm{s}$, consistent with the ion sound speed, but much too slow for electron sound speed.

Various frames from BES, ${ }^{16,24}$ taken at an arbitrary time, $t_{0}^{\mathrm{BES}}$ and various intervals later (up to $t_{0}^{\mathrm{BES}}+16 \mu \mathrm{s}$ ) and shown in Fig. 7, feature an ELM as a positive density feature appearing at the bottom of the frame and moving upward. The data shown are the deviation from the average value and in the color scheme, white represents average (or background) density, red and blue represent positive and negative fluctuations, and black indicates saturation. The LCFS is indicated by a solid vertical line. As time progresses, it is seen that the ELM appears at the top frame [Fig. 7(a)] and later, plasma ejections are produced near the separatrix [Fig. 7(c)] and move radially [Figs. 7(c)-7(e)]. The plasma ejection, marked with a circle, moves radially at nearly $8 \mathrm{~km} / \mathrm{s}$ in these discharges.

The data clearly indicate: (1) ELMs form in the low field side; (2) the ELMs have a finite poloidal and radial extent; (3) the ELM plasma moves radially at a few $\mathrm{km} / \mathrm{s}$ near the LCFS, (4) the ELM plasma also moves poloidally; and (5) the ELM plasma (or parts thereof) spreads radially as it moves towards the wall. The BES data were taken in a set of discharges different from the ones where probe data were taken and, thus the ELM detailed behavior may differ quantitatively; therefore, only the qualitative characteristics are emphasized.

A separate confirmation of the ELM radial propagation into the SOL, a reproducible feature, is obtained from reflectometry in a separate set of discharges. As the ELM evolves, the plasma travels outward as seen in Fig. 8(b), with a velocity that peaks at $\sim 500 \mathrm{~m} / \mathrm{s}$ and decays later as shown in Fig. 8(a). The velocity of the ELM plasma can be calculated from probe measurements as $V_{r}=E_{\theta} B / B^{2}$ and the data, marked as circles in Fig. 8(a), verify the reflectometry measured speed at the LCFS and its reduction with radius and time, slowing down to $\sim 120 \mathrm{~m} / \mathrm{s}$ near the wall.

A close look at fast diagnostic data, shown in Fig. 9, reveals that the ELMs have a complex spatiotemporal time structure, as reported recently, ${ }^{25}$ consistently with BES data, that can be observed as multiple spikes in the signals, particularly in the $I_{\text {sat }}$ current signal [Fig. 9(b)] from the midplane Langmuir reciprocating probe and the current from the floor tile array [Fig. 9(e)], which are the largest bandwidth
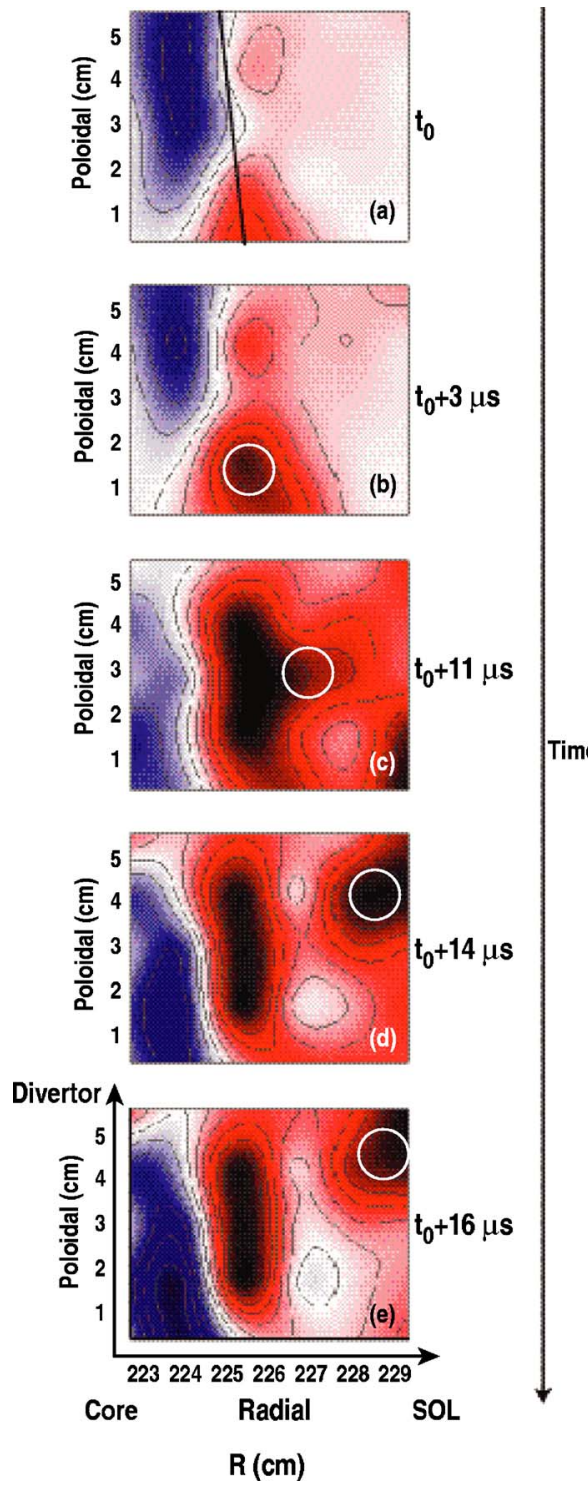

FIG. 7. Frames from BES showing 2-D density plots which were taken every $1 \mu \mathrm{s}$. The type I ELM starts at $t_{0}$ and the frames are taken at key times thereafter to illustrate ELM characteristics. The ELM shows a poloidal structure that quickly becomes quite complex. Radial and poloidal motion of ejection is clearly seen.

instruments considered. The data are somewhat smoother in the photodiode signals [Figs. 9(a), 9(c), and 9(d)], partially because of bandwidth and partially because of the surface and atomic physics involved. Although the ELMs are not completely reproducible, showing a scattering in amplitude of 20\% to 30\%, as seen in Figs. 9 and 10, they are similar enough that they can be assumed to be the same object for many purposes. The probe data were taken during various probe plunges in identical conditions and each probe plunge can contain information about several ELMs as seen in Figs. 9(b) and 10 (b). The probe is moving slowly ( 2 to $5 \mathrm{~m} / \mathrm{s}$ ) as compared to the ELMs [Fig. 10(a)], so it can be considered stationary for each ELM, but it moves enough between ELMs that the sensors are located at a significantly different radial position and, therefore, data on radial ELM variation are obtained.

Temperature and density data taken by probes at three 


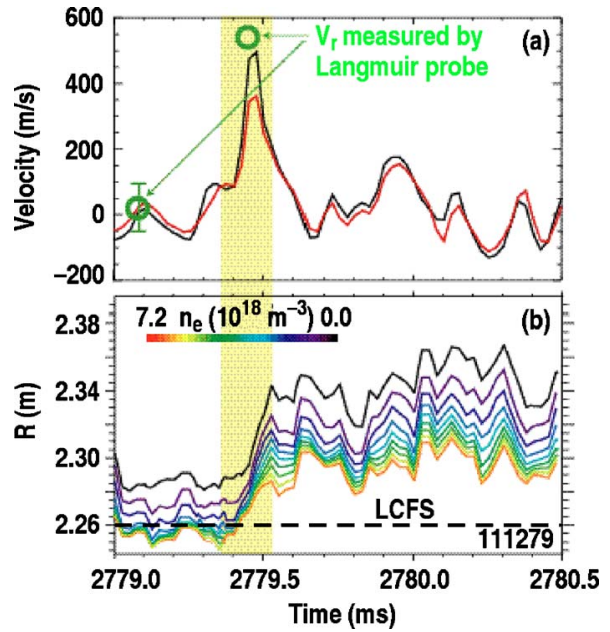

FIG. 8. Reflectometry data showing (a) velocity of the density front during an ELM and (b) contour plot of SOL density. The ELM moves rapidly from the LCFS at speeds up to $500 \mathrm{~m} / \mathrm{s}$ and reaches the wall within $200 \mu \mathrm{s}$. The reflectometry-measured density presented here reaches only $0.8 \times 10^{19} \mathrm{~m}^{-3}$ so only the lower pedestal (SOL) is seen.

radial locations in the SOL as ELMs travel radially past the comparatively slow-moving probe are shown in Fig. 11. It is important to notice that the different radial locations correspond to different ELMs. The high time resolution data, displayed in a $12 \mathrm{~m} / \mathrm{s}$ window that arbitrarily spans the ELM duration, is shown as dots and the running average as a thick line. The ELM plasma features peak $n_{e}$ values corresponding to those at the top of the density pedestal $(\approx 8.5$ $\times 10^{19} \mathrm{~m}^{-3}$ ) if the spatial decay is accounted for (Figs. 9 and 2). The pedestal $T_{e}$ and $n_{e}$ profile from Thomson scattering corresponding to the discharge above is highlighted with a thick line in Fig. 12. The ELM plasma in the near SOL $(R$ $-R_{\text {sep }}=0.4$ in Fig. 11) features peak $n_{e}$ values corresponding to that in the pedestal at the position marked with the vertical line in Fig. 12, i.e., $n_{e}=6.5 \times 10^{19} \mathrm{~m}^{-3}$, thus suggesting that

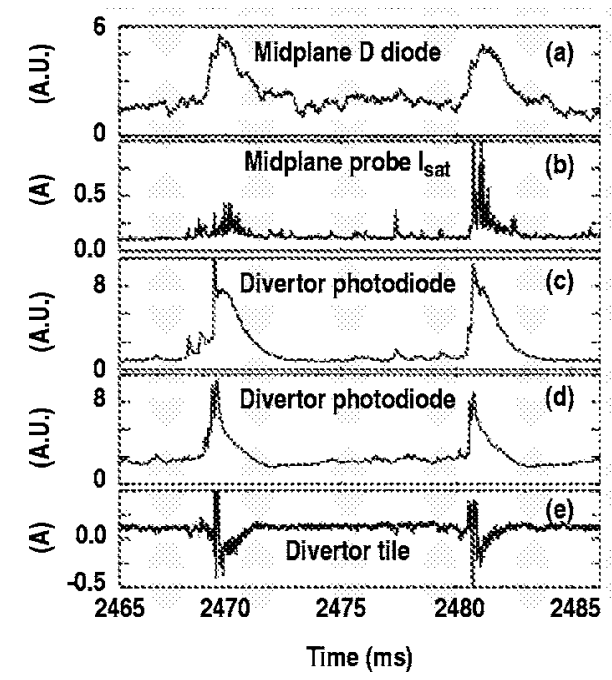

FIG. 9. Data from various fast diagnostics including (a) $D_{\alpha}$ from the midplane tangential array, (b) scanning probe $I_{\text {sat }}$, (c) and (d) $D_{\alpha}$ from the divertor array, and (e) current from the tile array. The complex structure of the ELMs is more evident in the fastest diagnostics.

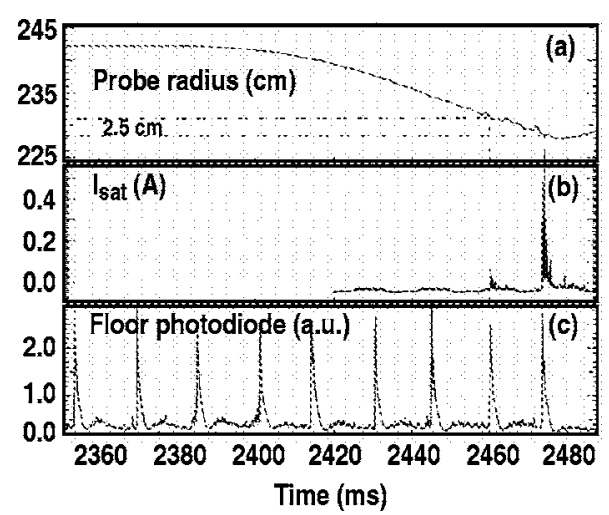

FIG. 10. The probe position versus time (a) is shown as two ELMs strike the probe sensors drawing a large saturation current, $I_{\text {sat }}$ (b) Since the ELMs are very reproducible (c), the variation in $I_{\text {sat }}$ is assumed to correspond to the radial attenuation of the ELMs.

the ELM peels off from the top of the density pedestal. The ELM plasma peak temperature $(\sim 225 \mathrm{eV})$ is lower than that at the indicated pedestal position $\left(T_{e} \sim 340 \mathrm{eV}\right)$, however, this discrepancy is due to the fact that the ELM plasma temperature has a decay length $(\sim 1 \mathrm{~cm})$ much shorter than the density decay length $(>2 \mathrm{~cm})$, as will be shown later. In summary, the data are consistent with the ELM plasma originating at the top of the density pedestal.

Inspection of the probe data in Fig. 11 leads to various immediate conclusions: (1) the data feature large bursts of $T_{e}$ and $n_{e}$, (2) the ELM carries plasma with density and temperature similar to those at the top of the density pedestal, (3) the peak density and temperature in the ELM decay with radius, (4) the temporal/spatial spread of the bursts seems to increase with radius, and (5) there is a quasistationary plasma background (thick line in Fig. 11) between the large spikes featuring a density of $\sim 2$ to $8 \times 10^{19} \mathrm{~m}^{-3}$ and temperature $\sim 5$ to $50 \mathrm{eV}$ (depending on radius).

In summary, this section presented evidence that: (1) ELMs originate in the low field side, (2) ELMs are comprised of short bursts of hot, dense plasma, (3) ELMs move
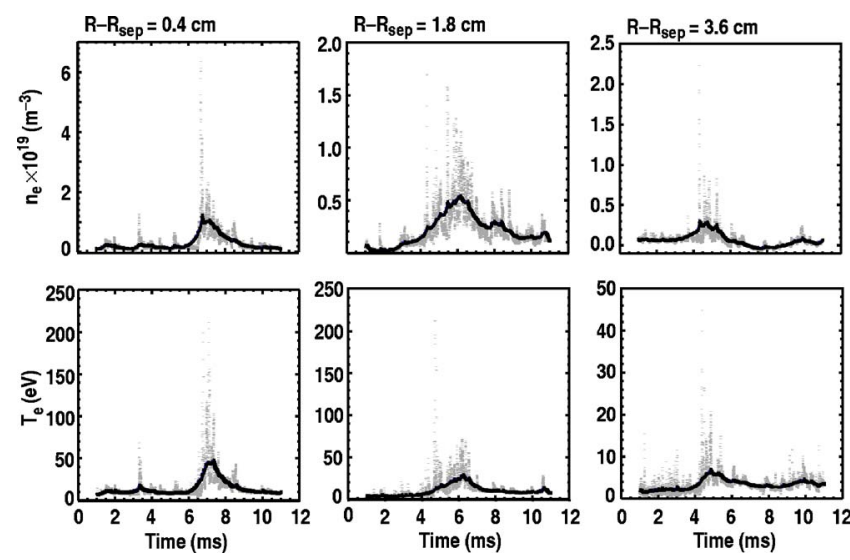

FIG. 11. High time resolution probe data of density (top row) and temperature (bottom row) for various radii showing the spatiotemporal complexity of ELMs and the loss of particles and energy as the ELM plasma leaves the LCFS behind. The relative timing of the ELM is arbitrary. 


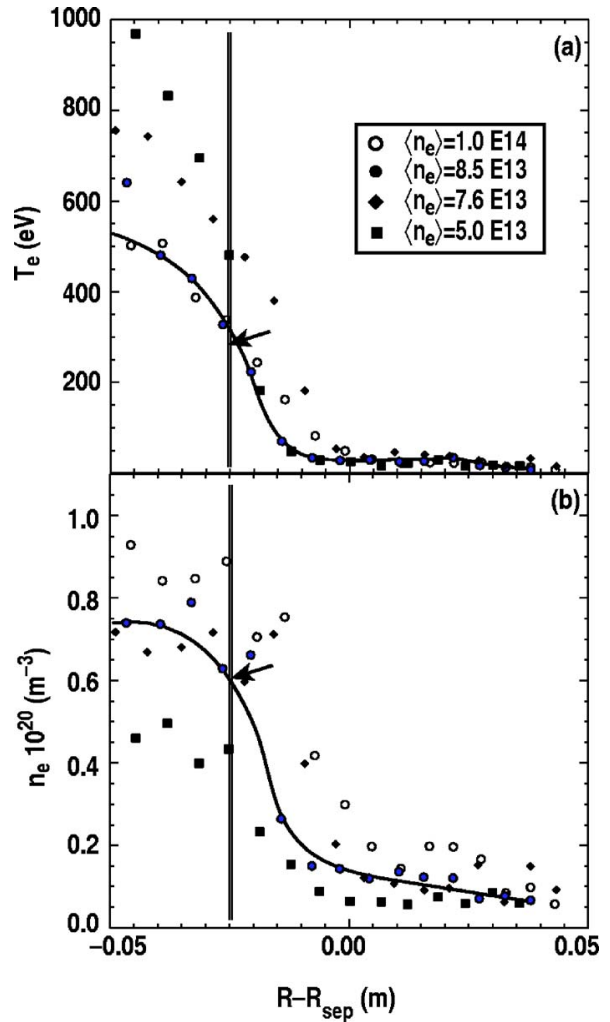

FIG. 12. Thomson scattering data featuring profiles of (a) temperature and (b) density in the plasma edge and SOL for discharges with various densities. The vertical line indicates the radius possessing $T_{e}$ and $N_{e}$ similar to the ELM peak values shown in Fig. 11. The pedestal profiles corresponding to the discharge with $\left\langle n_{e}\right\rangle=8.5 \times 10^{13} \mathrm{~cm}^{-3}$ are highlighted with a rough fit. The ELM carries with it information from that region in the pedestal, suggesting its birthplace.

radially and also poloidally (at $E \times B$ speed), (4) ELMs are probably originated at the top of the density pedestal, and (5) ELM bursts are poloidally localized.

\section{RADIAL PARTICLE AND HEAT ELM TRANSPORT}

Since issues of great concern related to ELM physics are those of recycling regions, impurity generation and transport and survivability of the walls and divertor, then a few questions seem immediate, namely: (1) how and how fast does the ELM plasma get to the wall, (2) how does the ELM plasma density and temperature decay with radius, and (3) how does the ELM plasma parameters scale with pedestal parameters?

From BES (Fig. 7) and probe data, it is clear that during the ELM there are various instances of plasma being ejected toward the SOL; these are clearly convective phenomena. The radial transport can be estimated in various ways; BES data shown in Fig. 7 indicate an initial velocity (at the LCFS) of $\sim 500$ to $1000 \mathrm{~m} / \mathrm{s}$; reflectometry data, shown in Fig. 8 indicate a peak ELM plasma velocity of $\sim 450 \mathrm{~m} / \mathrm{s}$ that rapidly slows down within $0.20 \mathrm{~m} / \mathrm{s}$, as the ELM crosses the SOL. Additionally, the ELM plasma features an internal (average) poloidal field, $E_{\theta}$, that is measured by two poloidally spaced tips and, therefore, the ELM radial speed in the SOL can be extracted as $V_{r}=\left(E_{\theta} \times B / B^{2}\right)$. The dataset described above yields ELM radial speeds of $\sim 450 \mathrm{~m} / \mathrm{s}$ near the sepa-

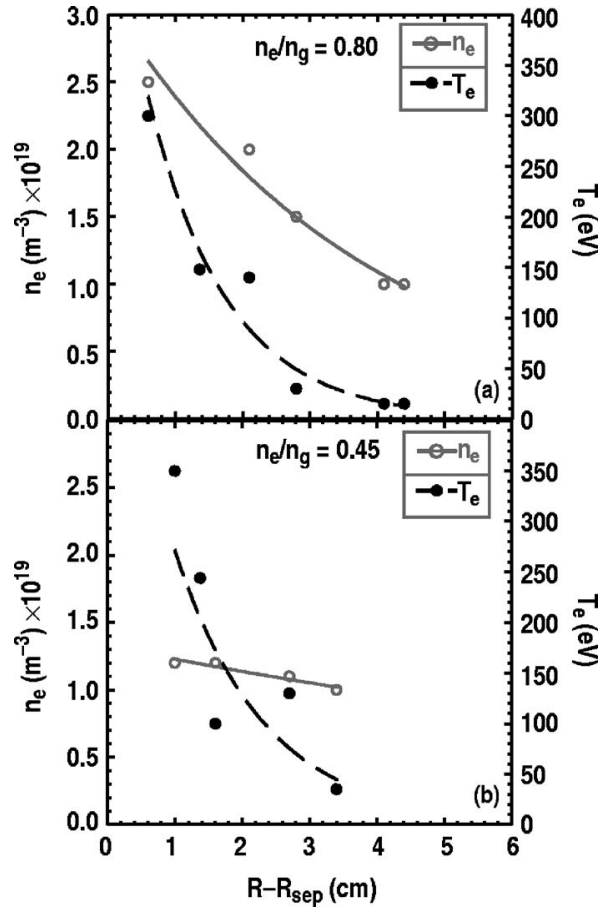

FIG. 13. Radial variation of the ELM peak density and temperature values obtained from probes for (a) high density and (b) low density discharges. The temperature decays quickly with radius in both cases, but the density decay length is much longer at low density.

ratrix that slow down to $\sim 120 \mathrm{~m} / \mathrm{s}$ at $4 \mathrm{~cm}$ into the $\mathrm{SOL}$ with a decay length of $\sim 1 \mathrm{~cm}$ and in fair agreement with the other measurements. At the highest speed, the ELM would strike the wall ( 6 to $7 \mathrm{~cm}$ from the LCFS) in $\sim 0.15 \mathrm{~m} / \mathrm{s}$, but due to the deceleration, the total radial transit time is closer to $0.3 \mathrm{~m} / \mathrm{s}$ for these discharges.

The peak density and temperature of the ELM plasma decay with radius as can be easily seen in Fig. 10 and is shown in detail in Fig. 13. A few conclusions can be immediately gained by inspecting the data: (1) the temperature falls with a short $(\sim 1.5 \mathrm{~cm})$ decay length, and (2) the density falls with a much longer ( $\sim 3$ to $10 \mathrm{~cm})$ decay length, indicating that the ELM heat is lost more efficiently (by parallel transport) than the particles. The density dependence will be discussed later.

It is possible to calculate the local peak heat and particle flux convected by the ELM toward the wall as $\Gamma_{r}=n V_{r}$ and $Q_{r}=2 \times 3 / 2 n T V_{r}$. Since, $T, n$, and $V_{r}$ decay with radius $\left(V_{r}\right.$ from $\sim 450$ to $\sim 150 \mathrm{~m} / \mathrm{s}$ ), an estimate can be made for the LCFS and another for the wall. Notice that the hot dense pulses of plasma exist on top of a less dense, colder plasma (Fig. 14) with $n_{e} \sim 5 \times 10^{18}, T_{e} \sim 50 \mathrm{eV}$ that also bears heat and particle fluxes to the wall, although much lower than the peak values; therefore, we will concentrate on peak values. The calculation assumes $T_{i}=T_{e}$ in the SOL, although Figs. 13 and 19 suggest that $\lambda_{T_{i}}>\lambda_{T_{e}}$, possibly leading to $T_{i}>T_{e}$ in the SOL and therefore, we could be underestimating the local heat flux. Unfortunately, probe data and CER data were taken in quite different discharges since the power had to be low for probe measurements resulting in too low CER signals. 


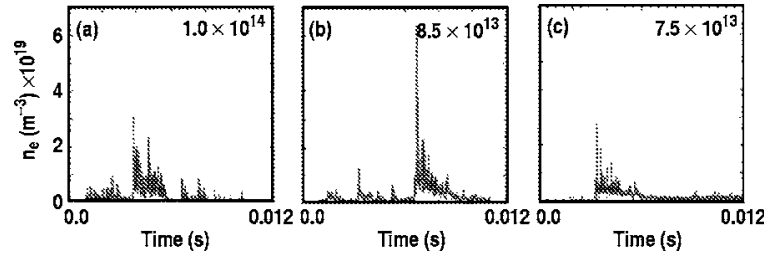

FIG. 14. Variation of the ELM density at $R-R_{\text {sep }} \sim 1 \mathrm{~cm}$ for (a) high density, (b) medium, and (c) low density discharges. The ELM density increases initially with discharge density but drops for the highest density case.

The measured ELM-induced convected radial heat flux at the LCFS due to the pulses at the wall (peak values) is $\sim 1800 \%$ of the average [calculated as $\left(P_{i n}-P_{r a d}\right) /$ Area, where $P_{\text {in }}-P_{\text {rad }} \sim 3.8 \mathrm{MW}$ and Area $\left.\sim 38 \mathrm{~m}^{2}\right]$ at the LCFS, at $\left\langle n_{e}\right\rangle / n_{G W}=0.8$ and $\sim 1500 \%$ at $\left\langle n_{e}\right\rangle / n_{G W}=0.45$. On the other hand, the pulsed power deposited by the ELM can be estimated by calculating the core energy losses during an ELM. $^{29}$ Such losses result in a loss of $\sim 0.0264 \mathrm{MJ}$ in approximately $0.5 \mathrm{~m} / \mathrm{s}$, resulting on average heat flux of $1.32 \mathrm{MW} / \mathrm{m}^{2}$ and quite comparable, within the approximations and error bars, to the LCFS heat flux values in Table I.

The convected local ELM-induced radial heat flux reaching the wall, shown in Table $\mathrm{I}$, is only a $\sim 2 \%$ fraction of the LCFS heat flux, consistently with the short $(\sim 1.5 \mathrm{~cm}) T_{e}$ radial decay. Thus, clearly much of the heat flux is transported along the magnetic field and strikes the divertor floor as has been previously observed by IR cameras. $^{26,27}$ which measure a broadening of the heat footprint during an ELM, thus supporting the measurements of radial heat transport.

On the other hand, the ELM local convected radial particle flux due to the pulses at the wall is $10 \%$ to $50 \%$ of the LCFS local radial flux, corresponding to a particle flux of $\sim 1.6 \times 10^{21} \mathrm{~m}^{-2} \mathrm{~s}^{-1}$, consistent with the long density decay length $(\sim 3$ to $8 \mathrm{~cm})$.

The ELM plasma peak temperature decay length is much shorter than the peak density decay length and the observation suggests that the physics that drains the ELMs of particles and energy is similar to that affecting bursty structures or blobs. The decay of the ELM (considered as elongated structures along the magnetic field) density and temperature can then be grossly predicted ${ }^{28}$ to be

TABLE I. Radial particle $\left(T_{r}^{E L M}\right)$ and heat $\left(Q_{r}^{E L M}\right)$ fluxes due to an ELM at the LCFS and the wall for low density $\left\langle n_{e}\right\rangle / n_{G W}=0.45$ high density $\left\langle n_{e}\right\rangle / n_{G W}=0.8$ discharges.

\begin{tabular}{ccc}
\hline \hline$\left\langle n_{e}\right\rangle / n_{G W}=0.8$ & $\Gamma_{r}^{E L M}\left(\mathrm{~m}^{-2} \mathrm{~s}^{-1}\right)$ & $Q_{r}^{E L M}\left(J \mathrm{~m}^{-2} \mathrm{~s}^{-1}\right)$ \\
LCFS & $1.0 \times 10^{22}$ & 1800000 \\
Wall & $1.5 \times 10^{21}$ & 21600 \\
& $\Gamma_{r}^{E L M}\left(\mathrm{~m}^{-2} \mathrm{~s}^{-1}\right)$ & $Q_{r}^{E L M}\left(J^{-2} \mathrm{~s}^{-1}\right)$ \\
$\left\langle n_{e}\right\rangle / n_{G W}=0.45$ & $5.6 \times 10^{21}$ & 1323000 \\
LCFS & $1.8 \times 10^{21}$ & 27000 \\
Wall & & \\
\hline
\end{tabular}

$$
n(r, t)=n(r) /\left(1+t / \tau_{T}\right)^{2 \alpha / \alpha_{T}} \quad \text { and } \quad T(t)=T_{0} /\left(1+t / \tau_{T}\right)^{2} \text {, }
$$

where $\alpha_{T}=\alpha S_{E} ; \tau_{T}=2 /\left(\alpha_{T} T_{0}^{1 / 2}\right), \alpha$ measures the net parallel current to the divertor plates, $S_{E}$ is the sheath energy transmission factor and $T_{0}$ is the temperature of the ELM as it detaches from the LCFS. Since $2 \alpha / \alpha_{T}=2 / S_{E} \ll 1$ and thus much smaller than the temperature exponent, the temperature decays much more rapidly than the density, just as observed in the experiment.

This section showed that (1) ELM plasma bursts convect (i.e., nondiffusive) heat and particles into the SOL, (2) the temperature has a short decay length (indicating dominance of parallel heat transport), (3) the density has a long decay length (indicating fast radial convective transport), and (4) heat and particle transport occur in short $(\sim 40 \mu \mathrm{s})$ bursts of hot, dense plasma on top of a quasistationary ( $\sim 1$ to $2 \mathrm{~m} / \mathrm{s}$ ), less dense, colder plasma transient.

\section{DENSITY SCALING OF ELM PLASMA AND TRANSPORT}

Since operating regimes that produce low amplitude, less damaging ELMs are being explored ${ }^{5,29,30}$ as a possible scenario for future machines, it is useful to investigate how the ELM plasma dynamics in the SOL varies with pedestal parameters. In these experiments, the density was increased on a discharge-to-discharge basis from $\left\langle n_{e}\right\rangle / n_{G W}=0.4$ to 0.8 .

The ELM density measured by the probe at $\sim 1 \mathrm{~cm}$ from the LCFS is shown in Fig. 14 for three different densities corresponding to $\left\langle n_{e}\right\rangle / n_{G W}=0.4,0.65$ and 0.8 . The most noticeable result is that the amplitude of the ELM density bursts increases with density but then drops at the highest density while the ELM time evolution also seems to change. The drop in ELM particle and energy content with density, due to a transition toward type III ELMs at higher collissionality, has been previously documented ${ }^{29}$ based on core plasma particle and energy balances.

The radial decay of the ELM peak density and temperature with distance from the LCFS varies with density as shown in Fig. 13. At high $n_{e}\left(n_{e} / n_{g}=0.85\right)$ the density decay length, $L_{N} \sim 3.8 \mathrm{~cm}$ while the temperature decay length, $L_{T}$ is $\sim 1.2 \mathrm{~cm}$. At $n_{e} / n_{g}=0.45, L_{N}$ and $L_{T}$ are $13 \mathrm{~cm}$ and $1.3 \mathrm{~cm}$, respectively. Therefore, at low densities, the particles in the ELM travel essentially unhindered toward the wall. While at high density, the particles are quickly drained or dissipated. The heat in the ELM seems to be dissipated rapidly with radius, no matter the pedestal density.

Additional information extracted from Table I is that the ELM local peak particle flux at the wall is quite similar for all densities due to the fact that at higher pedestal densities the ELM plasma density increases but the decay length becomes shorter.

DISRAD2 ELM data were studied during discharges (110394-110409) with densities at the edge of the pedestal ranging from $n_{e}=4 \times 10^{13}$ to $8 \times 10^{13} \mathrm{~cm}^{-3}$, with corresponding electron temperatures $T_{e}=300-600 \mathrm{eV}$. The results [Figs. 15(a) and 15(b)] indicate that radiation from the inner divertor increases with density and dominates at all densities, 

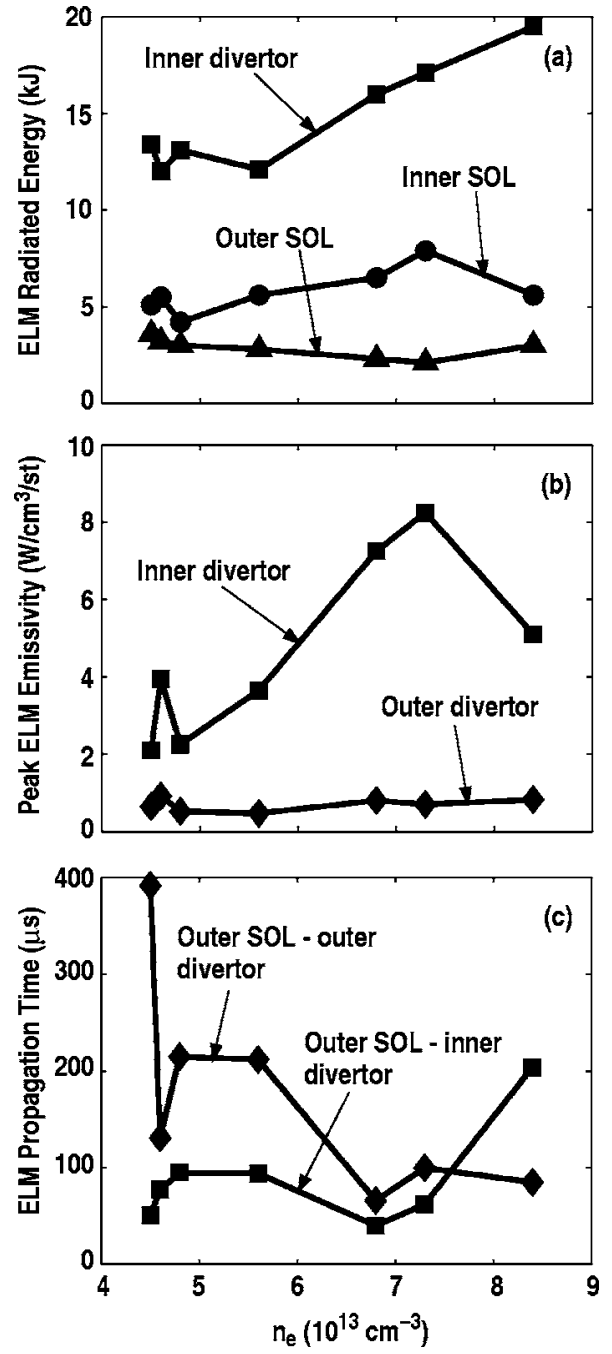

FIG. 15. Average values of (a) ELM radiated energy, (b) ELM peak emissivity, and (c) ELM propagation time as a function of plasma density for the various boundary regions marked in Fig. 4 as measured by DISRAD.

followed by that from the inner SOL, outer SOL, and outer divertor. The lag time between the surge of emissivity in the outer SOL (near where ELMs are produced) and the surge in the inner and outer divertor are shown in Fig. 15(c). Surprisingly, the lag is much larger between the outer SOL and the outer divertor than between the outer SOL and the inner divertor, but only at the lowest densities since they become roughly equal as the density increases. These observations can show a different behavior than that of the D-alpha radiation or particle fluxes by themselves. Since the radiation processes are complex, the distribution of impurities is involved, and the ELM motion may be quite complex; as seen later, interpretation is still open.

\section{VARIOUS INTERPRETATIONS OF THE ELM DYNAMICS DATA}

The data presented above offer new information about the properties of ELMs but have to be now reconciled with data from other diagnostics and to the current experimental and theoretical understanding. The ELMs are expected to start as poloidally, and perhaps even toroidally, localized.
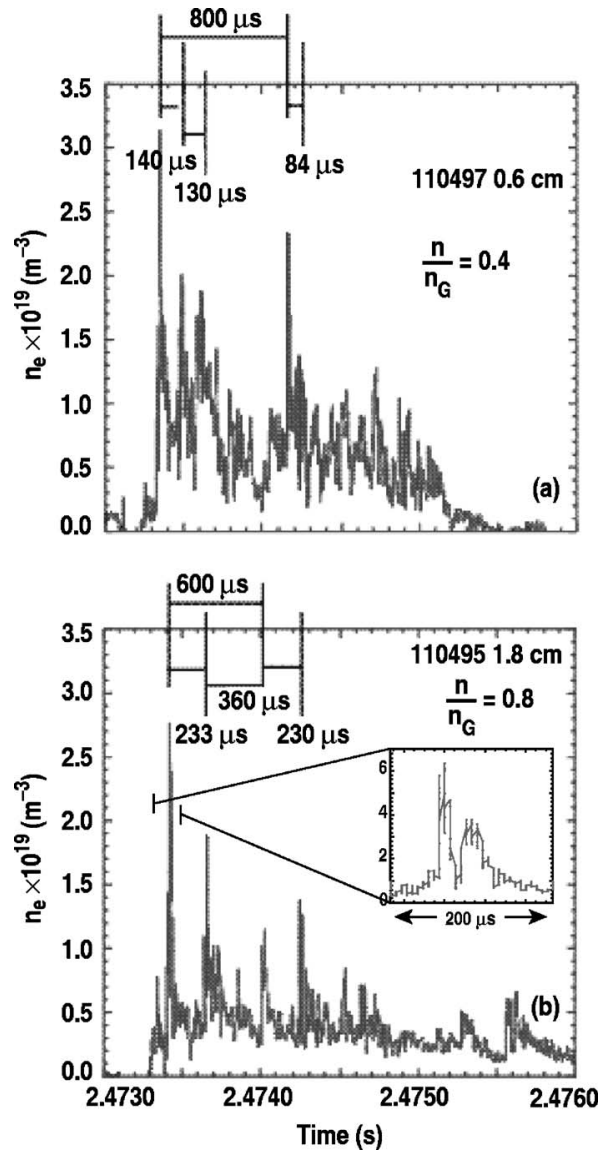

FIG. 16. High time-resolution ELM density data for (a) low discharge density $\left(n / n_{g}=0.4\right)$ and (b) high discharge density and $\left(n / n_{g}=0.8\right)$, illustrating the complex structure of ELMs. The successive bursts of high density lasts $\sim 30 \mu$ s [see inset in Fig. 16(b)] and initially appear at fairly regular intervals $(\sim 140 \mu$ s at high density, $230 \mu$ s at low density). The intervals can show some significant gaps of $\sim 300$ to $400 \mu$ s between groups of bursts.

Once the magnetic surfaces burst and plasma is spilled into the edge/SOL, a rapid expansion along the field lines is expected, together with radial motion. Among the many puzzles are (1) why supposedly poloidally localized events can be seen at essentially all poloidal and toroidal locations with little (100 to $200 \mu \mathrm{s})$ delay, comparable to ion midplane-divertor parallel transit times, (2) how can the complex structure of the fast signals be explained, and (3) how is the radial speed of the ELMs and corresponding lifetime $(\sim 400 \mu \mathrm{s})$ compatible with other time constants?

\section{A. Radial motion paradigm}

It is seen in Figs. 11 and 14 that the ELM features a high-density pulse propagating first and quickly followed by subsequent fronts of progressively decaying amplitude. Assuming mainly radial motion of the ELMs and using the measured radial ELM velocity, $V_{r}$, at the LCFS $(\sim 450 \mathrm{~m} / \mathrm{s})$, the radial extent of each front can be estimated as $\delta r=V_{r} \times \delta t$, where $\delta t$ is the duration of the pulse. Radial extents of 3 to $5 \mathrm{~cm}$ and of 2 to $3 \mathrm{~cm}$ per pulse are obtained from the pulse duration ( $\sim 20$ to $40 \mu$ s long) in Fig. 16 for the high $\left(n / n_{G}=0.8\right)$ and low $\left(n / n_{G}=0.4\right)$ density cases, respectively. Gaps lasting $\sim 130$ to $140 \mu$ s are seen between 


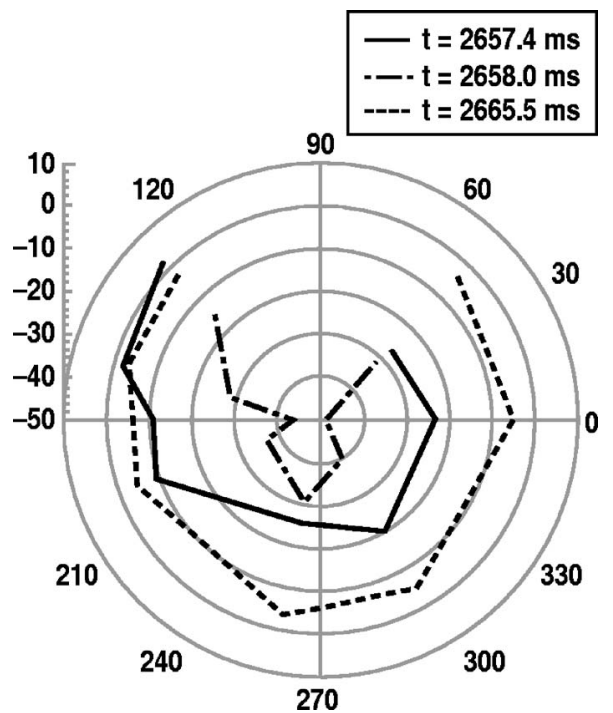

FIG. 17. Current from the tile array as function of toroidal angle (or tile position) for a time interval between Elms (dashed line) and two time slices during an ELM (solid line and dot-dashed line). During an ELM, toroidal asymmetries arise and change with time.

pulses in Fig. 16(a), sometimes followed by a longer gap of $\sim 800 \mu$ s between trains of pulses. The pulses are more regular in Fig. 16(b), with gaps of $\sim 230 \mu$ s followed by a longer gap of $\sim 360 \mu \mathrm{s}$. Therefore, the ELM comes from the LCFS in well-defined bursts that substantially fill the SOL (6 to 7 $\mathrm{cm}$ gap) with dense plasma. This paradigm, however, is hard to reconcile with the fact that many diagnostics in all toroidal and poloidal locations see the ELM with little time delay $(\sim 100 \mu \mathrm{s})$. The usual answer is that the ELMs have a high $n$ number in the linear phase and that a substantial number of these high amplitude regions will grow and nonlinearly expand simultaneously into the SOL, thus covering a large amount of the SOL volume.

Another possibility is that the ELM plasma is also rotating toroidally. A first piece of evidence is obtained from data from the tile array, shown in Fig. 17. The tile currents are plotted versus toroidal angle for two times during an ELM (solid lines) and compared to data in between ELMs (dashed line). It is clear that the tile toroidal current distribution, fairly symmetric before the ELM, develops an asymmetry that evolves in time during the ELM, as first presented by Evans $;^{18}$ however, these data are only supportive, but not conclusive of ELM toroidal motion.

Additional support for a rotating ELM paradigm is obtained from time-lag correlation calculations applied to the tile array data, shown in Fig. 18(a) and magnetic coil data, shown in Fig. 18(e). The correlation function is shown fitted by a Gaussian so that the time lag can be accurately measured as a fit parameter. The two signals used for the calculation and a photodiode signal for reference are plotted in each figure. The delay between the tile current signals is $96 \mu$ s during discharge 110496, and since the tiles are $45^{\circ}$ apart at $R=1.65 \mathrm{~m}$ radius, it corresponds to a toroidal velocity of $13.5 \mathrm{~km} / \mathrm{s}$. This velocity compares reasonably with the carbon toroidal speed of $22 \mathrm{~km} / \mathrm{s}$ at the LCFS measured us-
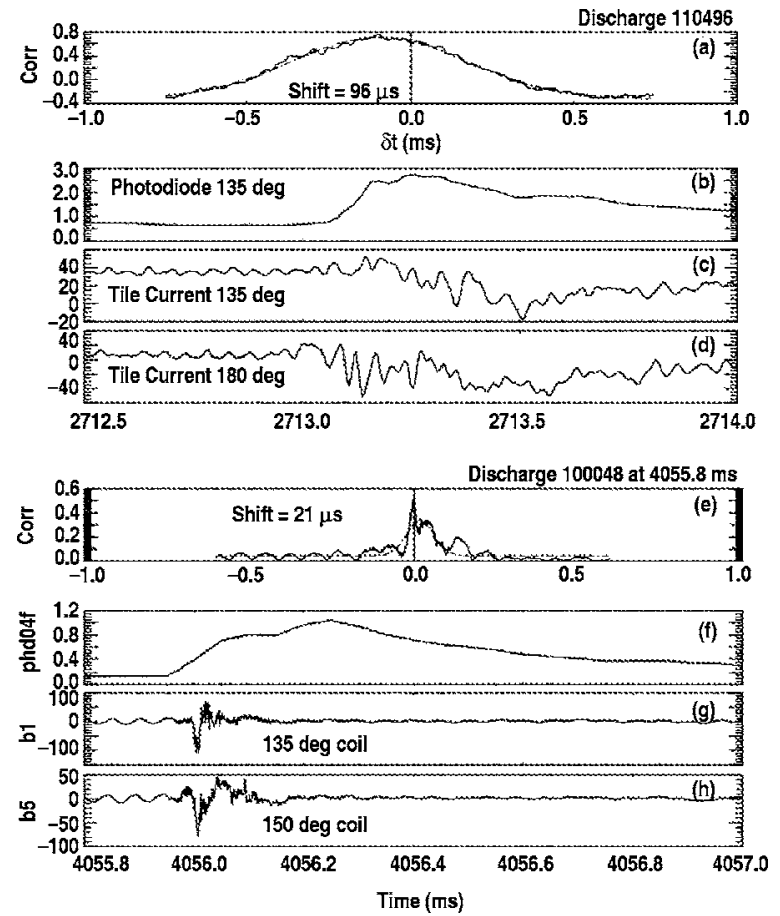

FIG. 18. Time lag cross correlation between signals from two floor tiles (a) is shown versus lag time indicating a delay of $90 \mu \mathrm{s}$. A floor $D_{\alpha}$ signal (b) is shown as a fiducial to compare to the two tile signals (c),(d). Similar calculation is performed for two B-dot coils indicating a lag of $21 \mu \mathrm{s}$ (e),(i).

ing $\mathrm{CER}^{31}$ for the same discharge. However, the CER measure carbon ion velocity and not background plasma velocity.

The photodiode signal featuring a single ELM and the CER speeds at radii near the LCFS are shown in Fig. 19, together with a rotation profile before $\left(t_{0}-1 \mathrm{~m} / \mathrm{s}\right)$ and during $\left(t_{0}\right)$ an ELM, where $t_{0}$ is determined by the rise of the fast $D_{\alpha}$ signal in the divertor and the floating potential in the midplane probe. The CER signals indicate a sudden drop in the edge/SOL carbon ion toroidal velocity during an ELM and the subsequent recovery $\sim 3 \mathrm{~m} / \mathrm{s}$ later, corresponding to momentum transport from the core. The sudden deceleration at the onset of an ELM in the innermost chord $\left(R-R_{\text {sep }}\right.$ $=-2.2 \mathrm{~cm}$ ) is concomitant with a transient increase further out and in the SOL, which lasts $\ll 1 \mathrm{~ms}$, consistent with the radial ELM plasma travel time $(\sim 0.3 \mathrm{~ms})$. Furthermore, the peak velocity of the radial transient is that of the innermost chord pre-ELM velocity. It is then reasonable to assume that the ELM carries some momentum with it upon detachment from the LCFS and that momentum keeps the ELM plasma rotating. The ELM plasma rotation velocity quickly slows down with radius in the SOL as seen in the rotation profile inset [Fig 19(b)]. The ion temperature, also shown in Fig. 19(c), increases steeply during the ELM to values close to the one at the top of the pedestal, supporting the idea of hot, dense plasma originating there. However, $T_{i}$ from CER cannot be compared to $T_{e}$ from probe measurements because the data have been taken in different discharge types due to diagnostic limitations. Closure of $T_{i}$ vs $T_{e}$ in the SOL question is an ongoing work and will be addressed in a future paper.

The CER-measured toroidal velocity is $\sim 27 \mathrm{~km} / \mathrm{s}$ in the SOL, corresponds to $\sim 650 \mu$ s period, longer than the ex- 


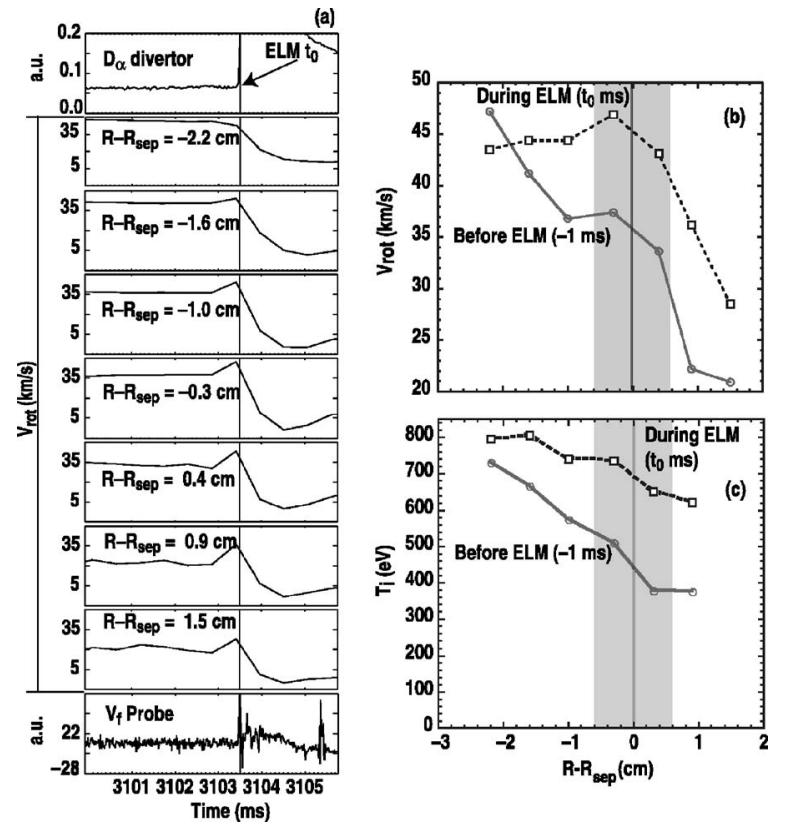

FIG. 19. (a) CER data showing the toroidal rotation velocity of carbon ions for seven radial positions inside the LCFS, and in the SOL. A floor $D_{\alpha}$ signal [(a) top] is used as a fiducial. Profiles of rotation velocity (b), an ion temperature (c) taken during the ELM $(t=0)$ and before the ELM $(t=-1)$ support the idea that the plasma at the top of the density pedestal is expelled into the SOL and rotates toroidally in the near SOL before being damped in the far SOL.

pected ELM plasma radial travel time of 200 to $300 \mu$ s, so the ELM plasma does not have time to rotate fully before striking the wall.

Once the rotation assumption is considered, Figs. 11, 14, and 17 and particularly Fig. 16, can be examined in a different light. The initial bursts in Fig. 16(a) are separated by $\sim 135 \mu$ s, followed by a $\sim 450 \mu$ s gap before the bursts start again. This sequence could be interpreted as three filaments rotating by the probe and returning with a period $T$ $=800 \mu$ s while decaying by parallel transport drainage. Likewise, Fig. 16(b) can be interpreted as two filaments returning after $\sim 600 \mu \mathrm{s}$.

This paradigm could address why the ELM signals always appear in all diagnostics within $200 \mu$ s or so and with relative delays that are hard to unravel. But more toroidally spread fast diagnostics are needed in DIII-D and other tokamaks.

\section{SUMMARY AND CONCLUSIONS}

Data from the DISRAD radiometer and BES clearly indicate ELM formation in the low field side of DIII-D and further propagation elsewhere. The BES data clearly show finite poloidal and radial extent of the ELM and, in agreement with reflectometry and probes, radial propagation. It is found that the ELMs feature complex, bursty plasma structures with $T_{e}$ and $N_{e}$ that can be traced to those at the top of the density pedestal, suggesting formation in that region. An implication of these data is that the modeling of ELM plasma particle and heat fluxes to the divertor is bursty in character, instead of a single pulse, with transient values much higher than the average. ${ }^{3,32}$

The radial propagation of coherent structures results in strong convection for particles and not so strong for heat. While the convective radial heat transport seems to be strongly dependent on density, that of particles is less so. An immediate implication of these data is that modelers should simulate ELM plasma transport as a convective process and not as a diffusive one. The bursty behavior of transport may also be important to modeling of heat flux to the divertor. ${ }^{3,32}$

The slow radial decay of the ELM plasma density indicates that resulting wall recycling and impurity release during ELMs will need to be investigated in more detail. The fast radial decay of the ELM plasma temperature suggests that sophisticated techniques will be necessary to mitigate the heat load to the divertor, which is now considered borderline for ITER. ${ }^{3}$

The data presented are in agreement with linear predictions of ELMS being strongly localized poloidally and arising in the pedestal. However, data indicate that the nonlinear phase of ELM evolution is key to some of its dynamical properties such as final volume, shape and radial velocity that determine the final impact of the ELMs on the walls and PFCs. Finally, the role of toroidal rotation on the ELM dynamics needs to be investigated further with fast, toroidally spread diagnostics.

\section{ACKNOWLEDGMENTS}

The technical support of L. Chousal and R. Hernandez are acknowledged.

This research was supported by the U.S. Department of Energy under Contract Nos. DE-FC02-04ER54698W-7405ENG-48DE-AC05-76OR00033DE-AC04-94AL85000 and Grant Nos. DE-FG03-95ER54294DE-FG03-96ER54373DEFG03-01ER54615DE-AC04-94AL8500.

${ }^{1}$ H. D. Pacher et al., ITER Design Description Document, ITER No. G 17 DDD 1 96-08-21 W2.1 (1996).

${ }^{2}$ A. W. Leonard, A. Herrmann, K. Itami, J. Lingertat, A. Loarte, T. H. Osborne, and W. Suttrop, J. Nucl. Mater. 266-269, 109 (1999).

${ }^{3}$ G. Federici, P. Andrew, P. Barabaschi et al., J. Nucl. Mater. 313-316 (2003).

${ }^{4}$ A. Loarte, G. Saibene, R. Sartori et al., J. Nucl. Mater. 313-316, 962 (2003).

${ }^{5}$ A. W. Leonard, T. H. Osborne, M. E. Fenstermacher, C. J. Lasnier, and M. A. Mahdavi, J. Nucl. Mater. 290-293, 1097 (2001).

${ }^{6}$ H. Urano, W. Suttrop, L. Horton et al., Plasma Phys. Controlled Fusion 45, 1571 (2003).

${ }^{7}$ A. Loarte, G. Saibene, R. Sartori et al., Plasma Phys. Controlled Fusion 45, 1549 (2003)

${ }^{8}$ J. W. Connor, R. J. Hastie, H. R. Wilson, and R. L. Miller, Phys. Plasmas 5, 2687 (1998).

${ }^{9}$ P. B. Snyder, H. R. Wilson, J. R. Ferron, L. L. Lao, A. W. Leonard, T. H. Osborne, A. D. Turnbull, D. Mossessian, M. Murakami, and X. Q. Xu, Phys. Plasmas 9, 2037 (2002).

${ }^{10}$ P. B. Snyder, H. R. Wilson, J. R. Ferron et al., "ELMs and constraints on the H-mode pedestal-A model based on peeling-ballooning modes," Proceedings of the 19th IAEA Fusion Energy Conference, Lyon, 2002 (International Atomic Energy Agency, Vienna, 2002) (to be published in Nucl. Fusion).

${ }^{11}$ H. R. Wilson, P. B. Snyder, G. T. Huysmans, and R. L. Miller, Phys. Plasmas 9, 1277 (2002).

${ }^{12}$ P. B. Snyder and X. Xu (private communication, 2003).

${ }^{13}$ J. L. Luxon, Nucl. Fusion 42, 614 (2002). 
${ }^{14}$ D. S. Gray, S. C. Luckhardt, A. G. Kellman et al., "Time resolved radiated power during tokamak disruptions and spectral averaging of AXUV photodiode response in DIII-D," Rev. Sci. Instrum. (to be published).

${ }^{15}$ J. Watkins, J. Salmonson, R. Moyer et al., Rev. Sci. Instrum. 63, 4728 (1992).

${ }^{16}$ R. J. Fonck, G. Cosby, R. D. Durst, S. F. Paul, N. Bretz, S. Scott, and E. Synakowski, Phys. Rev. Lett. 70, 3736 (1993).

${ }^{17}$ T. L. Rhodes, S. Baang, A. E. Chou, C. W. Domier, N. C. Luhmann, Jr., and W. A. Peebles, Rev. Sci. Instrum. 63, 4599 (1992).

${ }^{18}$ T. E. Evans, C. J. Lasnier, D. N. Hill et al., J. Nucl. Mater. 220-222, 235 (1995).

${ }^{19}$ D. L. Rudakov, J. A. Boedo, R. A. Moyer et al., Rev. Sci. Instrum. 72, 453 (2001).

${ }^{20}$ L. L. Lao, H. St. John, R. D. Stambaugh, A. G. Kellman, and W. Pfeiffer, Nucl. Fusion 25, 1611 (1985).

${ }^{21}$ L. Zeng, E. J. Doyle, T. L. Rhodes, G. Wang, W. A. Peebles, and K. H. Burrell, Rev. Sci. Instrum. 74, 1530 (2003).

${ }^{22}$ R. J. Colchin, D. L. Hillis, R. Maingi, C. C. Klepper, and N. H. Brooks, Rev. Sci. Instrum. 74, 2068 (2003).
${ }^{23}$ T. W. Petrie, J. G. Watkins, L. L. Lao, and P. B. Snyder, Nucl. Fusion 43, 1 (2003).

${ }^{24}$ G. R. McKee, R. Ashley, R. Durst, R. Fonck, M. Jakubowski, K. Tritz, K. Burrell, C. Greenfield, and J. Robinson, Rev. Sci. Instrum. 70, 913 (1999).

${ }^{25}$ B. Goncalves, C. Hidalgo, M. Pedrosa et al., Plasma Phys. Controlled Fusion 45, 1627 (2003).

${ }^{26}$ A. W. Leonard, J. A. Boedo, M. E. Fenstermacher et al., J. Nucl. Mater. 313-316, 768 (2003).

${ }^{27}$ D. N. Hill, R. Ellis, W. Ferguson, and D. E. Perkins, Rev. Sci. Instrum. 59, 1878 (1988)

${ }^{28}$ D. A. D'Ippolito, J. R. Myra, and S. I. Krasheninnikov, Phys. Plasmas 9, 222 (2002).

${ }^{29}$ M. E. Fenstermacher, A. W. Leonard, P. B. Snyder et al., Plasma Phys. Controlled Fusion 45, 1597 (2003).

${ }^{30}$ A. W. Leonard, T. H. Osborne, M. E. Fenstermacher et al., Phys. Plasmas 10, 1765 (2003).

${ }^{31}$ K. H. Burrell, Rev. Sci. Instrum. 72, 906 (2001).

${ }^{32}$ A. S. Kukushkin, H. D. Pacher, G. Federici, G. Janeschitz, A. Loarte, and G. W. Pacher, Fusion Eng. Des. 65, 355 (2003). 